\title{
ON PITERBARG THEOREM FOR MAXIMA OF STATIONARY GAUSSIAN SEQUENCES ${ }^{1}$
}

\section{Enkelejd Hashorva}

Department of Actuarial Science, Faculty of Business and Economics,University of Lausanne, UNIL-Dorigny 1015 Lausanne,

Switzerland

(e-mail: enkelejd.hashorva@unil.ch)

\section{Zuoxiang Peng}

School of Mathematics and Statistics, Southwest University, 400715 Chongqing, China

(e-mail: pzx@swu.edu.cn)

\section{Zhichao Weng}

Department of Actuarial Science, Faculty of Business and Economics,University of Lausanne, UNIL-Dorigny 1015 Lausanne,

Switzerland

(e-mail: zhichao.weng@unil.ch)

Received

Abstract. Limit distributions of maxima of dependent Gaussian sequence are different according to the convergence rate of their correlations. For three different conditions on convergence rate of the correlations, in this paper we establish Piterbarg theorem for maxima of stationary Gaussian sequences.

Keywords: ...Incomplete sample; Joint limit distribution; Maximum; Stationary Gaussian sequence; Weak and strong dependence; Piterbarg theorem.

AMS Classification: Primary 60G70; secondary 60G10

\section{INTRODUCTION}

For a strictly stationary sequence $\left\{X_{n}, n \geq 1\right\}$ the seminal paper [10] derived joint limiting distributions of maxima of complete and incomplete samples. The sample is often incomplete since observations are missing, which is formalized by introducing a sequence of indicator random variables $\left\{\varepsilon_{n}, n \geq 1\right\}$, where $\left\{\varepsilon_{n}=1\right\}$ means that $X_{n}$ is observed, whereas $\left\{\varepsilon_{n}=0\right\}$ corresponds to the case $X_{n}$ is missing. Throughout this paper $\left\{\varepsilon_{n}, n \geq 1\right\}$ are independent of the stationary process $\left\{X_{n}, n \geq 1\right\}$. If $F$ denote the common distribution function of all $X_{n}$ 's, then the maxima of incomplete sample $M_{n}(\varepsilon), n \geq 1$ is defined by

$$
M_{n}(\varepsilon)= \begin{cases}\max \left\{X_{j}, \varepsilon_{j}=1, j \leq n\right\}, & \text { if } \sum_{j=1}^{n} \varepsilon_{j} \geq 1, \\ \inf \{t: F(t)>0\}, & \text { otherwise }\end{cases}
$$

\footnotetext{
${ }^{1}$ E. Hashorva is supported partially by the Swiss National Science Foundation project 200021-1401633/1; Z. Weng has been partially supported by the Swiss National Science Foundation project 200021-134785 and by the project RARE -318984 (a Marie Curie International Research Staff Exchange Scheme Fellowship within the 7th European Community Framework Programme); Z. Peng has been supported by the National Natural Science Foundation of China under grant 11171275, the Natural Science Foundation Project of CQ under cstc2012jjA00029
} 
It is well-known (see e.g., [3]) that if for some norming constants $a_{n}>0$ and $b_{n} \in \mathbb{R}$

$$
\lim _{n \rightarrow \infty} F^{n}\left(a_{n} x+b_{n}\right)=G(x) \quad \forall x \in \mathbb{R}
$$

with $G$ being an extreme value distribution of some random variable $\mathcal{M}$, then

$$
\lim _{n \rightarrow \infty} \mathbb{P}\left\{a_{n}^{-1}\left(M_{n}-b_{n}\right) \leq x\right\}=G(x), \quad \forall x \in \mathbb{R}
$$

holds with $M_{n}=\max _{1 \leq i \leq n} X_{i}$ under some additional weak dependence conditions. Assume that for some constant $\mathcal{P} \in[0,1]$ the indicator random sequence $\left\{\varepsilon_{n}, n \geq 1\right\}$ satisfies

$$
\frac{S_{n}}{n}:=\frac{\sum_{i=1}^{n} \varepsilon_{i}}{n} \rightarrow \mathcal{P}, \text { in probability }
$$

as $n \rightarrow \infty$. The contribution [10] proved that under the conditions $D\left(u_{n}, v_{n}\right)$ and $D^{\prime}\left(u_{n}\right)$,

$$
\lim _{n \rightarrow \infty} \mathbb{P}\left\{M_{n}(\varepsilon) \leq u_{n}, M_{n} \leq v_{n}\right\}=H(\mathcal{P}, x, y)=: G^{\mathcal{P}}(x) G^{1-\mathcal{P}}(y)
$$

for $x<y$, where $u_{n}:=a_{n} x+b_{n}, v_{n}:=a_{n} y+b_{n}$; definitions of $D\left(u_{n}, v_{n}\right)$ and $D^{\prime}\left(u_{n}\right)$ can be found in [5], [7] and [10]. For the case that (1.3) holds with $\mathcal{P}$ a random variable, recently [5] showed that (1.4) still holds with $H(\mathcal{P}, x, y)=\mathbb{E}\left(G^{\mathcal{P}}(x) G^{1-\mathcal{P}}(y)\right)$.

A closely related work to [10] is contribution [6] which considers a strongly dependent stationary Gaussian random sequence $\left\{X_{n}, n \geq 1\right\}$ with correlation $r_{n}=\mathbb{E}\left(X_{1} X_{n+1}\right)$ such that

$$
\lim _{n \rightarrow \infty} r_{n} \ln n=\gamma \in[0, \infty)
$$

In this case $F=\Phi$ the distribution function of a $N(0,1)$ random variable, and therefore (1.1) holds with norming constants $a_{n}$ and $b_{n}$ given by

$$
a_{n}=\frac{1}{\sqrt{2 \ln n}}, \quad b_{n}=\sqrt{2 \ln n}-\frac{\ln \ln n+\ln 4 \pi}{2 \sqrt{2 \ln n}} .
$$

Assuming that (1.5) holds, we have (see [6],[9],[14])

$$
\lim _{n \rightarrow \infty} \mathbb{P}\left\{a_{n}^{-1}\left(M_{n}-b_{n}\right) \leq x\right\}=\mathbb{E}(\exp (-\exp (-x-\gamma+\sqrt{2 \gamma} W))),
$$

where $W$ is a $N(0,1)$ random variable. Clearly, when $\gamma=0$, the limit distribution in the right-hand side of (1.7) is the Gumbel distribution $\Lambda(x)=\exp (-\exp (-x)), x \in \mathbb{R}$ which is shown in the seminal paper [1]. Commonly the case $\gamma=0$ is referred to as the case of weak dependence, since the limit distribution of the maxima of the stationary process is the same as that of an iid sequence with underlying distribution function $\Phi$.

We expect that for this case, again (1.4) holds under the general settings of [5], which is confirmed in the next section. If $\gamma>0$, the limiting distribution of the maxima is a mixture distribution different from the Gumbel distribution, and thus for that case we cannot use the result of [5]. In order to overcome this difficulty we shall borrow some ideas from [6] which cover the strong dependence case.

In the seminal paper [13] V.I. Piterbarg considered the joint approximation of the maximum of a stationary Gaussian process over a discrete and continuous grid of points. The results in [6] and [10] are motivated by the ideas and techniques developed in the aforementioned paper. Therefore, we shall refer in this contribution to the joint limit distribution of maxima of complete and incomplete samples such as (1.4) as Piterbarg theorem.

In this paper we are concerned only with stationary Gaussian sequences assuming that (1.5) holds with $\gamma \in[0, \infty]$. For the case of a weakly dependent stationary Gaussian sequence $\left\{X_{n}, n \geq 1\right\}$, i.e., condition (1.5) (or the so-called 
Berman condition) holds with $\gamma=0$, then both conditions $D\left(u_{n}, v_{n}\right)$ and $D^{\prime}\left(u_{n}\right)$ hold with the choice of constants $a_{n}, b_{n}$ given by (1.6). Therefore in view of [5] Piterbarg theorem is valid (see Section2).

Our main results show that Piterbarg theorem also holds for the general case that $\gamma \in[0, \infty]$. Furthermore, we generalize the recent findings of [16] which are motivated by [6]. For some related work on asymptotic behavior of extremes of Gaussian sequences see [2, 11].

Brief organization of the rest of the paper: Section 2 presents the main results, their proofs are relegated to Section 3.

\section{MAIN RESULTS}

In the sequel, let $\left\{X_{n}, n \geq 1\right\}$ be a standard stationary Gaussian sequence with underlying distribution function $\Phi$ and correlations $\left\{r_{n}, n \geq 1\right\}$ satisfying the dependence condition (1.5) with $\gamma \in[0, \infty]$. As in the Introduction in order to derive Piterbarg theorem we shall assume further that the indicator random variables $\left\{\varepsilon_{n}, n \geq 1\right\}$ are independent of the Gaussian sequence and further (1.3) holds. Our first result is closely related to the result of [5].

Theorem 2.1 Suppose that the stationary Gaussian sequence $\left\{X_{n}, n \geq 1\right\}$ is independent of indicator sequence $\left\{\varepsilon_{n}, n \geq\right.$ $1\}$. If further (1.3) holds with some random variable $\mathcal{P}$, then under the Berman condition

$$
\lim _{n \rightarrow \infty} \mathbb{P}\left\{M_{n}(\varepsilon) \leq a_{n} x+b_{n}, M_{n} \leq a_{n} y+b_{n}\right\}=\mathbb{E}\left(\Lambda^{\mathcal{P}}(x) \Lambda^{1-\mathcal{P}}(y)\right)
$$

holds for all real $x, y$ with $x<y$ and constants $a_{n}$ and $b_{n}$ given by (1.6).

Note in passing that the Berman condition implies the convergence of sample maxima $\left\{M_{n}, n \geq 1\right\}$ (after normalisation) to a unit Gumbel random variable, whereas the above result implies that

$$
\lim _{n \rightarrow \infty} \mathbb{P}\left\{M_{n}(\varepsilon) \leq a_{n} x+b_{n}\right\}=\mathbb{E}\left(\Lambda^{\mathcal{P}}(x)\right), \quad \forall x \in \mathbb{R}
$$

and thus we have the joint convergence in distribution

$$
\left(\frac{M_{n}(\varepsilon)-b_{n}}{a_{n}}, \frac{M_{n}-b_{n}}{a_{n}}\right) \stackrel{d}{\rightarrow}(\widetilde{\mathcal{M}}, \mathcal{M}), \quad n \rightarrow \infty
$$

where $(\widetilde{\mathcal{M}}, \mathcal{M})$ have joint distribution function $H(\mathcal{P}, x, y)$ which is defined by $H(\mathcal{P}, x, y)=\mathbb{E}\left(\Lambda^{\mathcal{P}}(x) \Lambda^{1-\mathcal{P}}(y)\right)$ if $x<y$ and $H(\mathcal{P}, x, y)=\Lambda(y)$ otherwise.

In [6] and later [16] derived the limit of $\mathbb{P}\left\{M_{n}-M_{n}(\varepsilon) \leq a_{n} x, M_{n}(\varepsilon)-b_{n} \leq a_{n} y\right\}$ as $n \rightarrow \infty$ for any $x>0$ and $y \in \mathbb{R}$. By continuous mapping theorem, the joint convergence in (2.1) implies thus the following corollary which generalizes Theorem 1, Corollary 1, and Theorem 2 in [16].

Corollary 2.2 Under the assumptions of Theorem 2.1, we have the joint convergence in distribution

$$
\left(\frac{M_{n}-M_{n}(\varepsilon)}{a_{n}}, \frac{M_{n}(\varepsilon)-b_{n}}{a_{n}}\right) \stackrel{d}{\rightarrow}(\mathcal{M}-\widetilde{\mathcal{M}}, \widetilde{\mathcal{M}}), \quad n \rightarrow \infty .
$$

Next we consider strongly dependent Gaussian sequences.

Theorem 2.3 Suppose that the stationary Gaussian sequence $\left\{X_{n}, n \geq 1\right\}$ is independent of indicator sequence $\left\{\varepsilon_{n}, n \geq\right.$ $1\}$ and (1.3) holds with some random variable $\mathcal{P}$. If further (1.5) holds with $\gamma \in(0, \infty)$, then for all real $x, y$ with $x<y$, we have

$$
\lim _{n \rightarrow \infty} \mathbb{P}\left\{M_{n}(\varepsilon) \leq a_{n} x+b_{n}, M_{n} \leq a_{n} y+b_{n}\right\}=H(\mathcal{P}, x, y)
$$


where $a_{n}$ and $b_{n}$ are given by (1.6) and

$$
H(\mathcal{P}, x, y)=\mathbb{E}\left(\int_{-\infty}^{+\infty} \exp \left(-\mathcal{P} e^{-x-\gamma+\sqrt{2 \gamma} z}-(1-\mathcal{P}) e^{-y-\gamma+\sqrt{2 \gamma} z}\right) d \Phi(z)\right)
$$

Clearly, the above result can be stated as a joint convergence in distribution, i.e., we have again that (2.1) holds with $(\widetilde{\mathcal{M}}, \mathcal{M})$ which has joint distribution function $H(\mathcal{P}, x, y)$ given by $(2.3)$ for all $x<y$ and $H(\mathcal{P}, x, y)$ equals the right-hand side of (1.7) for $x \geq y$. Consequently, we obtain the following result which extends Theorem 3 in [16] where $\mathcal{P}$ is considered to be a constant.

Corollary 2.4 Under the assumptions of Theorem 2.3, the joint convergence in distribution in (2.2) holds where $(\widetilde{\mathcal{M}}, \mathcal{M})$ has the joint distribution function $H(\mathcal{P}, x, y)$ given by (2.3).

Remark 1. In view of [6] (see also [12]), the condition (1.5) can be slightly relaxed in the case that $\gamma \in(0, \infty)$. The result of Theorem 2.3 is still valid under the weaker condition stated in Eq. (5) in [6].

It is possible to have a joint convergence of sample maxima and that of incomplete sample maxima even if condition $D^{\prime}\left(u_{n}\right)$ is not satisfied see [10]. As shown therein for a stationary non-Gaussian process related to the storage process, both maxima are completely dependent, which is a result expected in view of the findings of [4].

Theorem 2.5 below shows that the case for strongly dependent Gaussian sequences satisfying (1.5) with $\gamma=\infty$. We recall first the result known in the literature for the convergence of the sample maxima. Namely, under the following two conditions

$$
\begin{aligned}
& r_{n} \text { is convex with } r_{n}=o(1) \text { and } \\
& \left(r_{n} \ln n\right)^{-1} \text { is monotone with }\left(r_{n} \ln n\right)^{-1}=o(1)
\end{aligned}
$$

as $n \rightarrow \infty$, [8] and [9] showed that with $\widetilde{b}_{n}:=\left(1-r_{n}\right)^{\frac{1}{2}} b_{n}$

$$
\lim _{n \rightarrow \infty} \mathbb{P}\left\{M_{n}-\widetilde{b}_{n} \leq \sqrt{r_{n}} x\right\}=\Phi(x), \quad \forall x \in \mathbb{R} .
$$

An extension of (2.6) to Piterbarg max-discretisation theorem is given in Corollary 2.2 of [15].

Our last result extends the above convergence as follows:

Theorem 2.5 Suppose that the stationary Gaussian sequence $\left\{X_{n}, n \geq 1\right\}$ is independent of indicator sequence $\left\{\varepsilon_{n}, n \geq\right.$ $1\}$ and (1.3) is valid with some random variable $\mathcal{P} \in(0,1]$. If (2.4) and (2.5) hold, then for all real $x, y$ we have

$$
\lim _{n \rightarrow \infty} \mathbb{P}\left\{M_{n}(\varepsilon)-\widetilde{b}_{n} \leq \sqrt{r_{n}} x, M_{n}-\widetilde{b}_{n} \leq \sqrt{r_{n}} y\right\}=\Phi(\min (x, y)),
$$

where $\widetilde{b}_{n}:=\left(1-r_{n}\right)^{\frac{1}{2}} b_{n}$ with $b_{n}$ given by (1.6).

Again we can cast the above result in the framework of joint convergence in distribution stated in (2.1), namely

$$
\left(\frac{M_{n}(\varepsilon)-\widetilde{b}_{n}}{\sqrt{r_{n}}}, \frac{M_{n}-\widetilde{b}_{n}}{\sqrt{r_{n}}}\right) \stackrel{d}{\rightarrow} \quad(W, W), \quad n \rightarrow \infty,
$$

where $W$ has $N(0,1)$ distribution. Consequently, in the language of [6] we have

$$
\left(\frac{M_{n}(\varepsilon)-\widetilde{b}_{n}}{\sqrt{r_{n}}}, \frac{M_{n}-M_{n}(\varepsilon)}{\sqrt{r_{n}}}\right) \stackrel{d}{\rightarrow}(W, 0), \quad n \rightarrow \infty .
$$




\section{FURTHER RESULTS AND PROOFS}

In order to prove the main theorems we need some auxiliary results. We borrow the following notation from [5]. Let $u_{n}(x)=a_{n} x+b_{n}, x \in \mathbb{R}$ and $\alpha=\left\{\alpha_{n}, n \geq 1\right\}$ be a non-random sequence taking values in $\{0,1\}$. For fixed $k$ let

$$
K_{s}=\{j:(s-1) m+1 \leq j \leq s m\}, \quad 1 \leq s \leq k,
$$

where $m=[n / k]$. Further, for a random variable $\mathcal{P}$ such that $0 \leq \mathcal{P} \leq 1$ a.s., set

$$
B_{t, k}=\left\{\omega: \mathcal{P}(\omega) \in\left\{\begin{array}{ll}
{\left[0, \frac{1}{2^{k}}\right],} & t=0 \\
\left(\frac{t}{2^{k}}, \frac{t+1}{2^{k}}\right], & 0<t \leq 2^{k}-1
\end{array}\right\}\right\}
$$

and define

$$
B_{t, k, \alpha, n}=\left\{\omega: \varepsilon_{j}(\omega)=\alpha_{j}, 1 \leq j \leq n\right\} \cap B_{t, k}
$$

In the following $C$ is a positive constant, its value might change in different lines; we use thus $C$ to omit $O(1)$ notation.

Lemma 1. Let $\left\{X_{n}^{*}, n \geq 1\right\}$ be a sequence of independent standard Gaussian random variables. For large $n$, there exists a positive integer $l$ such that $k<l<m=[n / k]$ and $l=o(n)$, then for $x<y$

$\left|\mathbb{P}\left\{M_{n}^{*}(\alpha) \leq u_{n}(x), M_{n}^{*} \leq u_{n}(y)\right\}-\prod_{s=1}^{k} \mathbb{P}\left\{M^{*}\left(K_{s}, \alpha\right) \leq u_{n}(x), M^{*}\left(K_{s}\right) \leq u_{n}(y)\right\}\right| \leq(4 k+2) l\left(1-\Phi\left(u_{n}(x)\right)\right)$

holds uniformly for all $\alpha \in\{0,1\}^{n}$, where $M_{n}^{*}=\max \left\{X_{j}^{*}, 1 \leq j \leq n\right\}, M^{*}\left(K_{s}\right)=\max \left\{X_{j}^{*}, j \in K_{s}\right\}$,

$$
M^{*}\left(K_{s}, \alpha\right)= \begin{cases}\max \left\{X_{j}^{*}, \alpha_{j}=1, j \in K_{s}\right\}, & \text { if } \sum_{j \in K_{s}} \alpha_{j} \geq 1 \\ -\infty, & \text { otherwise }\end{cases}
$$

and

$$
M_{n}^{*}(\alpha)= \begin{cases}\max \left\{X_{j}^{*}, \alpha_{j}=1,1 \leq j \leq n\right\}, & \text { if } \sum_{j=1}^{n} \alpha_{j} \geq 1 \\ -\infty, & \text { otherwise }\end{cases}
$$

ProOf OF Lemma 1. First, we classify $k m$ integers into $2 k$ consecutive intervals as follows. For large $n$, let $l$ be an integer such that $k<l<m$ and $l=o(n)$. Write

$$
I_{s}=\{(s-1) m+1, \ldots, s m-l\}, \quad J_{s}=\{s m-l+1, \ldots, s m\}
$$

for $1 \leq s \leq k$, and set

$$
I_{k+1}=\{(k-1) m+l+1, \ldots, k m\}, \quad J_{k+1}=\{k m+1, \ldots, k m+l\} .
$$

Since $\left\{X_{n}^{*}, n \geq 1\right\}$ are independent, using the arguments similar to the proof of Lemma 4.3 in [10], we obtain the desired result.

Lemma 2. Under the conditions of Theorem 2.3 for $x<y$ we have

$$
\begin{aligned}
& \left|\mathbb{P}\left\{M_{n}(\alpha) \leq u_{n}(x), M_{n} \leq u_{n}(y)\right\}-\int_{-\infty}^{+\infty} \mathbb{P}\left\{M_{n}^{*}(\alpha) \leq v_{n}(x, z), M_{n}^{*} \leq v_{n}(y, z)\right\} d \Phi(z)\right| \\
& \quad \leq C n \sum_{k=1}^{n}\left|r_{k}-\rho_{n}\right| \exp \left(-\frac{u_{n}^{2}(x)}{1+w_{k}}\right)
\end{aligned}
$$


holds uniformly for all $\alpha \in\{0,1\}^{n}$, where $v_{n}(x, z)=\left(1-\rho_{n}\right)^{-\frac{1}{2}}\left(u_{n}(x)-\rho_{n}^{\frac{1}{2}} z\right), \rho_{n}=\gamma / \ln n, w_{k}=\max \left\{\left|r_{k}\right|, \rho_{n}\right\}$ and some positive constant $C$.

ProOf OF LEMmA 2. Let $\left\{\xi_{n, k}, 1 \leq k \leq n, n \geq 1\right\}$ be a triangular array of standard Gaussian random variables with equally correlation $\rho_{n}=\gamma / \ln n$, and define

$$
M_{n}^{\xi}(\alpha)= \begin{cases}\max \left\{\xi_{n, j}, \alpha_{j}=1,1 \leq j \leq n\right\}, & \text { if } \sum_{j=1}^{n} \alpha_{j} \geq 1 \\ -\infty, & \text { otherwise }\end{cases}
$$

and $M_{n}^{\xi}=\max \left\{\xi_{n, k}, 1 \leq k \leq n\right\}$. By Berman's inequality (see e.g., [7] or [12])

$$
\begin{aligned}
& \left|\mathbb{P}\left\{M_{n}(\alpha) \leq u_{n}(x), M_{n} \leq u_{n}(y)\right\}-\mathbb{P}\left\{M_{n}^{\xi}(\alpha) \leq u_{n}(x), M_{n}^{\xi} \leq u_{n}(y)\right\}\right| \\
\leq & C n \sum_{k=1}^{n}\left|r_{k}-\rho_{n}\right| \exp \left(-\frac{u_{n}^{2}(x)}{1+w_{k}}\right)
\end{aligned}
$$

holds uniformly for all $\alpha \in\{0,1\}^{n}$, where $w_{k}=\max \left\{\left|r_{k}\right|, \rho_{n}\right\}$. According to the proof of Theorem 6.5.1 in [7]

$$
\mathbb{P}\left\{M_{n}^{\xi}(\alpha) \leq u_{n}(x), M_{n}^{\xi} \leq u_{n}(y)\right\}=\int_{-\infty}^{+\infty} \mathbb{P}\left\{M_{n}^{*}(\alpha) \leq v_{n}(x, z), M_{n}^{*} \leq v_{n}(y, z)\right\} d \Phi(z),
$$

where $v_{n}(x, z)=\left(1-\rho_{n}\right)^{-\frac{1}{2}}\left(u_{n}(x)-\rho_{n}^{\frac{1}{2}} z\right)$ and thus the claim follows.

Lemma 3. Let $\left\{Y_{n, k}, 1 \leq k \leq n, n \geq 1\right\}$ be a triangular array of standard Gaussian sequences with correlation $\rho_{k}=$ $\left(r_{k}-r_{n}\right) /\left(1-r_{n}\right)$ where $r_{k}$ satisfies (2.4) and (2.5) for $k=1,2, \ldots, n$. Suppose that $\left\{Y_{n, k}, 1 \leq k \leq n, n \geq 1\right\}$ is independent of indicator sequence $\left\{\varepsilon_{n}, n \geq 1\right\}$. If further that (1.3) holds with some random variable $\mathcal{P} \in(0,1]$, then for all $\delta>0$ we have

$$
\lim _{n \rightarrow \infty} \mathbb{P}\left\{M_{n}^{Y}(\varepsilon) \leq b_{n}-\delta r_{n}^{\frac{1}{2}}\right\}=0
$$

where

$$
M_{n}^{Y}(\varepsilon)= \begin{cases}\max \left\{Y_{n, j}, \varepsilon_{j}=1,1 \leq j \leq n\right\}, & \text { if } \sum_{j=1}^{n} \varepsilon_{j} \geq 1 \\ -\infty, & \text { otherwise. }\end{cases}
$$

PROOF OF LEMmA 3. Let $\left\{Z_{n, k}, 1 \leq k \leq n, n \geq 1\right\},\left\{W_{n, k}, 1 \leq k \leq n, n \geq 1\right\}$ be two triangular arrays of standard Gaussian sequences with correlations defined by

$$
\mathbb{E}\left(Z_{n, 1} Z_{n, i+1}\right)=\left\{\begin{array}{ll}
\rho_{i}, & 1 \leq i \leq t(n), \\
\rho_{t(n)}, & i>t(n),
\end{array} \quad \mathbb{E}\left(W_{n, 1} W_{n, i+1}\right)=\sigma_{i}= \begin{cases}\frac{\rho_{i}-\rho_{t(n)},}{1-\rho_{t(n)}} & 1 \leq i \leq t(n), \\
0, & i>t(n),\end{cases}\right.
$$

respectively, where $t(n)=\left[n \exp \left(-(\ln n)^{1 / 2}\right)\right]$. Suppose that the two Gaussian sequences are independent of the indicator sequence $\left\{\varepsilon_{n}, n \geq 1\right\}$. Define similarly to above $M_{n}^{Z}(\varepsilon)$ and $M_{n}^{W}(\varepsilon)$ and let $\eta$ be a standard Gaussian random variable being independent of $\left\{W_{n, k}, 1 \leq k \leq n, n \geq 1\right\}$. Using Slepian's inequality (see e.g., [12]) we have

$$
\begin{aligned}
\mathbb{P}\left\{M_{n}^{Y}(\alpha) \leq b_{n}-\delta r_{n}^{\frac{1}{2}}\right\} & \leq \mathbb{P}\left\{M_{n}^{Z}(\alpha) \leq b_{n}-\delta r_{n}^{\frac{1}{2}}\right\} \\
& =\mathbb{P}\left\{\left(1-\rho_{t(n)}\right)^{\frac{1}{2}} M_{n}^{W}(\alpha)+\rho_{t(n)}^{\frac{1}{2}} \eta \leq b_{n}-\delta r_{n}^{\frac{1}{2}}\right\} \\
& =\int_{-\infty}^{+\infty} \mathbb{P}\left\{M_{n}^{W}(\alpha) \leq\left(b_{n}-\delta r_{n}^{\frac{1}{2}}-\rho_{t(n)}^{\frac{1}{2}} z\right)\left(1-\rho_{t(n)}\right)^{-\frac{1}{2}}\right\} d \Phi(z) \\
& \leq \Phi\left(-\delta r_{n}^{\frac{1}{2}} /\left(2 \rho_{t(n)}^{\frac{1}{2}}\right)\right)+\mathbb{P}\left\{M_{n}^{W}(\alpha) \leq\left(b_{n}-\delta r_{n}^{\frac{1}{2}} / 2\right)\left(1-\rho_{t(n)}\right)^{-\frac{1}{2}}\right\}
\end{aligned}
$$


Further, using Berman's inequality we obtain

$$
\begin{aligned}
& \left|\mathbb{P}\left\{M_{n}^{W}(\alpha) \leq\left(b_{n}-\delta r_{n}^{\frac{1}{2}} / 2\right)\left(1-\rho_{t(n)}\right)^{-\frac{1}{2}}\right\}-\mathbb{P}\left\{M_{n}^{*}(\alpha) \leq\left(b_{n}-\delta r_{n}^{\frac{1}{2}} / 2\right)\left(1-\rho_{t(n)}\right)^{-\frac{1}{2}}\right\}\right| \\
\leq & C n \sum_{i=1}^{t(n)} \sigma_{i} \exp \left(-\frac{\left(b_{n}-\delta r_{n}^{\frac{1}{2}} / 2\right)^{2}}{\left(1+\sigma_{i}\right)\left(1-\rho_{t(n)}\right)}\right)=: c_{n} .
\end{aligned}
$$

Hence by the total probability formula

$$
\begin{aligned}
\mathbb{P}\left\{M_{n}^{Y}(\varepsilon) \leq b_{n}-\delta r_{n}^{\frac{1}{2}}\right\} & =\sum_{t=0}^{2^{k}-1} \sum_{\alpha \in\{0,1\}^{n}} \mathbb{P}\left\{M_{n}^{Y}(\alpha) \leq b_{n}-\delta r_{n}^{\frac{1}{2}}\right\} \mathbb{P}\left(B_{t, k, \alpha, n}\right) \\
& \leq \Phi\left(-\delta r_{n}^{\frac{1}{2}} /\left(2 \rho_{t(n)}^{\frac{1}{2}}\right)\right)+\mathbb{P}\left\{M_{n}^{*}(\varepsilon) \leq\left(b_{n}-\delta r_{n}^{\frac{1}{2}} / 2\right)\left(1-\rho_{t(n)}\right)^{-\frac{1}{2}}\right\}+c_{n}
\end{aligned}
$$

By the properties (2.4) and (2.5) of $\left\{r_{n}, n \geq 1\right\}$, the following useful facts (see [9], page 9):

$$
\lim _{n \rightarrow \infty} \frac{r_{n}}{\rho_{t(n)}}=\infty, \quad \lim _{n \rightarrow \infty} \frac{b_{n} \rho_{t(n)}}{r_{n}^{1 / 2}}=0
$$

imply that

$$
\lim _{n \rightarrow \infty} \Phi\left(-\delta r_{n}^{\frac{1}{2}} /\left(2 \rho_{t(n)}^{\frac{1}{2}}\right)\right)=0
$$

and

$$
\mathbb{P}\left\{M_{n}^{*}(\varepsilon) \leq\left(b_{n}-\delta r_{n}^{\frac{1}{2}} / 2\right)\left(1-\rho_{t(n)}\right)^{-\frac{1}{2}}\right\} \leq \mathbb{P}\left\{M_{n}^{*}(\varepsilon) \leq-a_{n} A+b_{n}\right\}
$$

for arbitrary positive number $A$ as $n$ large. By Corollary 1 in [5]

$$
\limsup _{n \rightarrow \infty} \mathbb{P}\left\{M_{n}^{*}(\varepsilon) \leq\left(b_{n}-\delta r_{n}^{\frac{1}{2}} / 2\right)\left(1-\rho_{t(n)}\right)^{-\frac{1}{2}}\right\} \leq \mathbb{E}\left(\Lambda^{\mathcal{P}}(-A)\right)
$$

Letting $A \rightarrow \infty$, we have

$$
\lim _{n \rightarrow \infty} \mathbb{P}\left\{M_{n}^{*}(\varepsilon) \leq\left(b_{n}-\delta r_{n}^{\frac{1}{2}} / 2\right)\left(1-\rho_{t(n)}\right)^{-\frac{1}{2}}\right\}=0
$$

By the arguments of [8] p.187-188 we have that $\lim _{n \rightarrow \infty} c_{n}=0$, hence the claim follows.

Proof of THEOREM 2.1. For a stationary Gaussian sequence, the conditions $D\left(u_{n}, v_{n}\right)$ and $D^{\prime}\left(u_{n}\right)$ hold when the correlations satisfies (1.5) with $\gamma=0$, see Lemma 4.4.1 in [7] for details. Hence, according to Theorem 1.1 in [5], we obtain the desired result.

Proof of THEOREM 2.3. Let $\Psi(n, x, z)=n\left(1-\Phi\left(v_{n}(x, z)\right)\right.$ with $v_{n}(x, z)=\left(1-\rho_{n}\right)^{-\frac{1}{2}}\left(u_{n}(x)-\rho_{n}^{\frac{1}{2}} z\right)$. Note that

$$
\begin{aligned}
&\left|\mathbb{P}\left\{M_{n}(\varepsilon) \leq u_{n}(x), M_{n} \leq u_{n}(y)\right\}-\mathbb{E}\left(\int_{-\infty}^{+\infty} \prod_{s=1}^{k}\left(1-\frac{\mathcal{P} \Psi(n, x, z)+(1-\mathcal{P}) \Psi(n, y, z)}{k}\right) d \Phi(z)\right)\right| \\
& \leq \sum_{t=0}^{2^{k}-1} \sum_{\alpha \in\{0,1\}^{n}} \mathbb{E}\left(\mid \mathbb{P}\left\{M_{n}(\alpha) \leq u_{n}(x), M_{n} \leq u_{n}(y)\right\}\right. \\
&\left.\left.\quad-\int_{-\infty}^{+\infty} \prod_{s=1}^{k}\left(1-\frac{\mathcal{P} \Psi(n, x, z)+(1-\mathcal{P}) \Psi(n, y, z)}{k}\right) d \Phi(z)\right) \mid \mathbb{I}\left(B_{t, k, \alpha, n}\right)\right) \\
& \leq E_{1}+E_{2}+E_{3}+E_{4},
\end{aligned}
$$


where

$$
\begin{aligned}
& E_{1}=\sum_{t=0}^{2^{k}-1} \sum_{\alpha \in\{0,1\}^{n}} \mathbb{E}\left(\mid \mathbb{P}\left\{M_{n}(\alpha) \leq u_{n}(x), M_{n} \leq u_{n}(y)\right\}\right. \\
& \left.-\int_{-\infty}^{+\infty} \mathbb{P}\left\{M_{n}^{*}(\alpha) \leq v_{n}(x, z), M_{n}^{*} \leq v_{n}(y, z)\right\} d \Phi(z) \mid \mathbb{I}\left(B_{t, k, \alpha, n}\right)\right), \\
& E_{2}=\sum_{t=0}^{2^{k}-1} \sum_{\alpha \in\{0,1\}^{n}} \mathbb{E}\left(\int_{-\infty}^{+\infty} \mid \mathbb{P}\left\{M_{n}^{*}(\alpha) \leq v_{n}(x, z), M_{n}^{*} \leq v_{n}(y, z)\right\}\right. \\
& \left.-\prod_{s=1}^{k} \mathbb{P}\left\{M^{*}\left(K_{s}, \alpha\right) \leq v_{n}(x, z), M^{*}\left(K_{s}\right) \leq v_{n}(y, z)\right\} \mid d \Phi(z) \mathbb{I}\left(B_{t, k, \alpha, n}\right)\right), \\
& E_{3}=\sum_{t=0}^{2^{k}-1} \sum_{\alpha \in\{0,1\}^{n}} \mathbb{E}\left(\int_{-\infty}^{+\infty} \mid \prod_{s=1}^{k} \mathbb{P}\left\{M^{*}\left(K_{s}, \alpha\right) \leq v_{n}(x, z), M^{*}\left(K_{s}\right) \leq v_{n}(y, z)\right\}\right. \\
& \left.-\prod_{s=1}^{k}\left(1-\frac{\frac{t}{2^{k}} \Psi(n, x, z)+\left(1-\frac{t}{2^{k}}\right) \Psi(n, y, z)}{k}\right) \mid d \Phi(z) \mathbb{I}\left(B_{t, k, \alpha, n}\right)\right)
\end{aligned}
$$

and

$$
\begin{aligned}
E_{4}= & \sum_{t=0}^{2^{k}-1} \sum_{\alpha \in\{0,1\}^{n}} \mathbb{E}\left(\int_{-\infty}^{+\infty} \mid \prod_{s=1}^{k}\left(1-\frac{\mathcal{P} \Psi(n, x, z)+(1-\mathcal{P}) \Psi(n, y, z)}{k}\right)\right. \\
& \left.-\prod_{s=1}^{k}\left(1-\frac{\frac{t}{2^{k}} \Psi(n, x, z)+\left(1-\frac{t}{2^{k}}\right) \Psi(n, y, z)}{k}\right) \mid d \Phi(z) \mathbb{I}\left(B_{t, k, \alpha, n}\right)\right) .
\end{aligned}
$$

Using Lemma 2 and Lemma 6.4.1 in [7], we have

$$
E_{1} \leq C n \sum_{i=1}^{n}\left|r_{i}-\rho_{n}\right| \exp \left(-\frac{u_{n}^{2}(x)}{1+w_{i}}\right) \rightarrow 0
$$

as $n \rightarrow \infty$. For $E_{2}$, according to Lemma 1 , we have

$$
E_{2} \leq(4 k+2) \frac{l}{n} \int_{-\infty}^{+\infty} \Psi(n, x, z) d \Phi(z)
$$

According to the proof of Theorem 6.5.1 in [7], we have $v_{n}(x, z)=u_{n}(x+\gamma-\sqrt{2 \gamma} z)+o\left(a_{n}\right)$, thus

$$
\lim _{n \rightarrow \infty} \Psi(n, x, z)=\exp (-x-\gamma+\sqrt{2 \gamma} z)=: h(x, z, \gamma)
$$

Combing with $l=o(n)$ as $n \rightarrow \infty$ the dominated convergence theorem yields

$$
\lim _{n \rightarrow \infty} E_{2}=0
$$


Next, using Lemma 3 in [5], we have

$$
\begin{aligned}
& E_{3} \leq \sum_{t=0}^{2^{k}-1} \sum_{\alpha \in\{0,1\}^{n}} \mathbb{E}\left(\int_{-\infty}^{+\infty} \sum_{s=1}^{k} \mid \mathbb{P}\left\{M^{*}\left(K_{s}, \alpha\right) \leq v_{n}(x, z), M^{*}\left(K_{s}\right) \leq v_{n}(y, z)\right\}\right. \\
& \left.-\left(1-\frac{\frac{t}{2^{k}} \Psi(n, x, z)+\left(1-\frac{t}{2^{k}}\right) \Psi(n, y, z)}{k}\right) \mid d \Phi(z) \mathbb{I}\left(B_{t, k, \alpha, n}\right)\right) \\
& \leq \sum_{t=0}^{2^{k}-1} \sum_{\alpha \in\{0,1\}^{n}} \mathbb{E}\left(\int_{-\infty}^{+\infty} \sum_{s=1}^{k}\left|\frac{\sum_{j \in K_{s}} \alpha_{j}}{m}-\frac{t}{2^{k}}\right| \frac{n\left(\Phi\left(v_{n}(y, z)\right)-\Phi\left(v_{n}(x, z)\right)\right)}{k} d \Phi(z) \mathbb{I}\left(B_{t, k, \alpha, n}\right)\right) \\
& +\frac{1}{k} \int_{-\infty}^{+\infty}(\Psi(n, x, z))^{2} d \Phi(z) \\
& =\sum_{t=0}^{2^{k}-1} \sum_{s=1}^{k} \mathbb{E}\left(\left|\sum_{j \in K_{s}} \frac{\varepsilon_{j}}{m}-\frac{t}{2^{k}}\right| \mathbb{I}\left(B_{t, k}\right)\right) \int_{-\infty}^{+\infty} \frac{n\left(\Phi\left(v_{n}(y, z)\right)-\Phi\left(v_{n}(x, z)\right)\right)}{k} d \Phi(z) \\
& +\frac{1}{k} \int_{-\infty}^{+\infty}(\Psi(n, x, z))^{2} d \Phi(z) \\
& \leq \sum_{s=1}^{k}\left(\mathbb{E}\left|\sum_{j \in K_{s}} \frac{\varepsilon_{j}}{m}-\mathcal{P}\right|+\frac{1}{2^{k}}\right) \int_{-\infty}^{+\infty} \frac{n\left(\Phi\left(v_{n}(y, z)\right)-\Phi\left(v_{n}(x, z)\right)\right)}{k} d \Phi(z) \\
& +\frac{1}{k} \int_{-\infty}^{+\infty}(\Psi(n, x, z))^{2} d \Phi(z) \\
& \leq \sum_{s=1}^{k}\left[2(2 s-1)\left(d\left(\frac{S_{s m}}{s m}, \mathcal{P}\right)+d\left(\frac{S_{(s-1) m}}{(s-1) m}, \mathcal{P}\right)\right)+\frac{1}{2^{k}}\right] \int_{-\infty}^{+\infty} \frac{\Psi(n, x, z)-\Psi(n, y, z)}{k} d \Phi(z) \\
& +\frac{1}{k} \int_{-\infty}^{+\infty}(\Psi(n, x, z))^{2} d \Phi(z),
\end{aligned}
$$

where $d(X, Y)$ stands for Ky Fan metric, i.e., $d(X, Y)=\inf \{\epsilon: \mathbb{P}\{|X-Y|>\epsilon\}<\epsilon\}$. Since $\lim _{m \rightarrow \infty} d\left(\frac{S_{s m}}{s m}, \mathcal{P}\right)=$ 0 , taking a limit $n \rightarrow \infty$ and then $m \rightarrow \infty$ we obtain

$$
\limsup _{n \rightarrow \infty} E_{3} \leq \frac{1}{2^{k}} \int_{-\infty}^{+\infty}(h(x, z, \gamma)-h(y, z, \gamma)) d \Phi(z)+\frac{1}{k} \int_{-\infty}^{+\infty} h^{2}(x, z, \gamma) d \Phi(z)
$$

For $E_{4}$, we have

$$
\begin{aligned}
E_{4} & \leq \sum_{t=0}^{2^{k}-1} \sum_{\alpha \in\{0,1\}^{n}} \mathbb{E}\left(\int_{-\infty}^{+\infty} \sum_{s=1}^{k}\left|\mathcal{P}-\frac{t}{2^{k}}\right| \frac{\Psi(n, y, z)+\Psi(n, x, z)}{k} d \Phi(z) \mathbb{I}\left(B_{t, k, \alpha, n}\right)\right) \\
& =\int_{-\infty}^{+\infty}(\Psi(n, y, z)+\Psi(n, x, z)) d \Phi(z) \sum_{t=0}^{2^{k}-1} \mathbb{E}\left(\left|\mathcal{P}-\frac{t}{2^{k}}\right| \mathbb{I}\left(B_{t, k}\right)\right) \\
& \leq \int_{-\infty}^{+\infty} \frac{\Psi(n, y, z)+\Psi(n, x, z)}{2^{k}} d \Phi(z) \\
& \rightarrow \frac{1}{2^{k}} \int_{-\infty}^{+\infty}(h(x, z, \gamma)+h(y, z, \gamma)) d \Phi(z)
\end{aligned}
$$


as $n \rightarrow \infty$. Hence, combining with (3.1)-(3.4), we have

$$
\begin{aligned}
& \limsup _{n \rightarrow \infty} \mid \mathbb{P}\left\{M_{n}(\varepsilon) \leq u_{n}(x), M_{n} \leq u_{n}(y)\right\} \\
& \quad-\mathbb{E}\left(\int_{-\infty}^{+\infty}\left(1-\frac{\mathcal{P} \exp (-x-\gamma+\sqrt{2 \gamma} z)+(1-\mathcal{P}) \exp (-y-\gamma+\sqrt{2 \gamma} z)}{k}\right)^{k} d \Phi(z)\right) \mid \\
& \leq \frac{1}{2^{k-1}} \int_{-\infty}^{+\infty} h(x, z, \gamma) d \Phi(z)+\frac{1}{k} \int_{-\infty}^{+\infty} h^{2}(x, z, \gamma) d \Phi(z) .
\end{aligned}
$$

The claimed result follows by letting $k \rightarrow \infty$.

Proof OF THEOREM 2.5. We show next that

$$
\lim _{n \rightarrow \infty} \mathbb{P}\left\{r_{n}^{-\frac{1}{2}}\left(M_{n}(\varepsilon)-\left(1-r_{n}\right)^{\frac{1}{2}} b_{n}\right) \leq x\right\}=\Phi(x), \quad \forall x \in \mathbb{R}
$$

Let events $B_{t, k, \alpha, n}$ be defined as before. Since by the assumption $\mathcal{P}>0$ and the indicator random sequence $\left\{\varepsilon_{n}, n \geq 1\right\}$ is independent of $\left\{X_{n}, n \geq 1\right\}$ for any $x \in \mathbb{R}$ we have

$$
\mathbb{P}\left\{r_{n}^{-\frac{1}{2}}\left(M_{n}(\varepsilon)-\left(1-r_{n}\right)^{\frac{1}{2}} b_{n}\right) \leq x\right\}=\sum_{t=0}^{2^{k}-1} \sum_{\alpha \in\{0,1\}^{n}} P(n, \alpha) \mathbb{P}\left(B_{t, k, \alpha, n}\right),
$$

where $P(n, \alpha)=\mathbb{P}\left\{r_{n}^{-\frac{1}{2}}\left(M_{n}(\alpha)-\left(1-r_{n}\right)^{\frac{1}{2}} b_{n}\right) \leq x\right\}$. Applying Slepian's inequality, we have further

$$
\begin{aligned}
P(n, \alpha) & =\int_{-\infty}^{+\infty} \mathbb{P}\left\{M_{n}^{Y}(\alpha) \leq b_{n}+r_{n}^{\frac{1}{2}}\left(1-r_{n}\right)^{-\frac{1}{2}}(x-z)\right\} d \Phi(z) \\
& \geq \int_{-\infty}^{+\infty} \mathbb{P}\left\{M_{n}^{*}(\alpha) \leq b_{n}+r_{n}^{\frac{1}{2}}\left(1-r_{n}\right)^{-\frac{1}{2}}(x-z)\right\} d \Phi(z) \\
& \geq \mathbb{P}\left\{M_{n}^{*}(\alpha) \leq b_{n}+r_{n}^{\frac{1}{2}}\left(1-r_{n}\right)^{-\frac{1}{2}} \delta\right\} \Phi(x-\delta)
\end{aligned}
$$

for any $\delta>0$. Since $\lim _{n \rightarrow \infty} a_{n}^{-1} r_{n}^{1 / 2}=\infty$, there exists sufficiently large $A$ such that for all $n$ large

$$
\begin{aligned}
\sum_{t=0}^{2^{k}-1} \sum_{\alpha \in\{0,1\}^{n}} P(n, \alpha) \mathbb{P}\left(B_{t, k, \alpha, n}\right) & \geq \Phi(x-\delta) \sum_{t=0}^{2^{k}-1} \sum_{\alpha \in\{0,1\}^{n}} \mathbb{P}\left\{M_{n}^{*}(\alpha) \leq b_{n}+r_{n}^{\frac{1}{2}}\left(1-r_{n}\right)^{-\frac{1}{2}} \delta\right\} \mathbb{P}\left(B_{t, k, \alpha, n}\right) \\
& =\Phi(x-\delta) \mathbb{P}\left\{M_{n}^{*}(\varepsilon) \leq b_{n}+r_{n}^{\frac{1}{2}}\left(1-r_{n}\right)^{-\frac{1}{2}} \delta\right\} \\
& \geq \Phi(x-\delta) \mathbb{P}\left\{M_{n}^{*}(\varepsilon) \leq b_{n}+a_{n} A \delta\right\} \\
& \geq \Phi(x-\delta) \mathbb{P}\left\{M_{n}^{*} \leq b_{n}+a_{n} A \delta\right\} .
\end{aligned}
$$

Clearly, since

$$
\lim _{A \rightarrow \infty} \lim _{n \rightarrow \infty} \mathbb{P}\left\{M_{n}^{*} \leq b_{n}+a_{n} A \delta\right\}=\lim _{A \rightarrow \infty} \exp (-\exp (-A \delta))=1
$$

we have

$$
\liminf _{n \rightarrow \infty} \mathbb{P}\left\{r_{n}^{-\frac{1}{2}}\left(M_{n}(\varepsilon)-\left(1-r_{n}\right)^{\frac{1}{2}} b_{n}\right) \leq x\right\} \geq \Phi(x-\delta)
$$


Next, we derive the upper bound. Note that

$$
\begin{aligned}
P(n, \alpha) & \leq \int_{-\infty}^{+\infty} \mathbb{P}\left\{M_{n}^{Y}(\alpha) \leq b_{n}+r_{n}^{\frac{1}{2}}\left(1-r_{n}\right)^{-\frac{1}{2}}(x-z)\right\} d \Phi(z) \\
& \leq \Phi(x+\delta)+\mathbb{P}\left\{M_{n}^{Y}(\alpha) \leq b_{n}-r_{n}^{\frac{1}{2}}\left(1-r_{n}\right)^{-\frac{1}{2}} \delta\right\}
\end{aligned}
$$

implying

$$
\mathbb{P}\left\{r_{n}^{-\frac{1}{2}}\left(M_{n}(\varepsilon)-\left(1-r_{n}\right)^{\frac{1}{2}} b_{n}\right) \leq x\right\} \leq \Phi(x+\delta)+\mathbb{P}\left\{M_{n}^{Y}(\varepsilon) \leq b_{n}-r_{n}^{\frac{1}{2}}\left(1-r_{n}\right)^{-\frac{1}{2}} \delta\right\} .
$$

Using Lemma 3 we obtain

$$
\limsup _{n \rightarrow \infty} \mathbb{P}\left\{r_{n}^{-\frac{1}{2}}\left(M_{n}(\varepsilon)-\left(1-r_{n}\right)^{\frac{1}{2}} b_{n}\right) \leq x\right\} \leq \Phi(x+\delta)
$$

hence (3.5) follows by letting $\delta \downarrow 0$. Next note that for any $x, y$

$p_{n}(x, y):=\mathbb{P}\left\{r_{n}^{-\frac{1}{2}}\left(M_{n}(\varepsilon)-\left(1-r_{n}\right)^{\frac{1}{2}} b_{n}\right) \leq x, r_{n}^{-\frac{1}{2}}\left(M_{n}-\left(1-r_{n}\right)^{\frac{1}{2}} b_{n}\right) \leq y\right\} \leq \mathbb{P}\left\{r_{n}^{-\frac{1}{2}}\left(M_{n}(\varepsilon)-\left(1-r_{n}\right)^{\frac{1}{2}} b_{n}\right) \leq x\right\}$

and further for $x<y$

$$
\begin{aligned}
\mathbb{P} & \left\{r_{n}^{-\frac{1}{2}}\left(M_{n}(\varepsilon)-\left(1-r_{n}\right)^{\frac{1}{2}} b_{n}\right) \leq x\right\} \\
& \leq p_{n}(x, y)+\mathbb{P}\left\{r_{n}^{-\frac{1}{2}}\left(M_{n}(\varepsilon)-\left(1-r_{n}\right)^{\frac{1}{2}} b_{n}\right) \leq y\right\}-\mathbb{P}\left\{r_{n}^{-\frac{1}{2}}\left(M_{n}-\left(1-r_{n}\right)^{\frac{1}{2}} b_{n}\right) \leq y\right\} \\
& =p_{n}(x, y)+o(1),
\end{aligned}
$$

where the last claim above follows directly by the fact that (see (2.6))

$$
\lim _{n \rightarrow \infty} \mathbb{P}\left\{r_{n}^{-\frac{1}{2}}\left(M_{n}-\left(1-r_{n}\right)^{\frac{1}{2}} b_{n}\right) \leq x\right\}=\Phi(x), \quad \forall x \in \mathbb{R} .
$$

Consequently, for $x<y$ we have $p_{n}(x, y)=\mathbb{P}\left\{r_{n}^{-\frac{1}{2}}\left(M_{n}(\varepsilon)-\left(1-r_{n}\right)^{\frac{1}{2}} b_{n}\right) \leq x\right\}+o(1)$ and thus the proof is complete.

\section{REFERENCES}

1. S.M. Berman, Limit theorems for the maximum term in stationary sequences, Ann. Math. Statist., 35:502-516, 1964.

2. L. Cao and Z. Peng, Asymptotic distributions of maxima of complete and incomplete samples from strongly dependent stationary Gaussian sequences, Appl. Math. Lett., 24(2):243-247, 2011.

3. M. Falk, J. Hüsler, and R.-D. Reiss, Laws of Small Numbers: Extremes and Rare Events, in DMV Seminar, Volume 23, p. 3rd edn, Birkhäuser, Basel, 2010.

4. J. Hüsler and V.I. Piterbarg, Limit theorem for maximum of the storage process with fractional Brownian motion as input, Stochastic Process. Appl., 114(2):231-250, 2004.

5. T. Krajka, The asymptotic behaviour of maxima of complete and incomplete samples from stationary sequences, Stochastic Process. Appl., 121(8):1705-1719, 2011.

6. A.V. Kudrov and V.I. Piterbarg, On maxima of partial samples in Gaussian sequences with pseudo-stationary trends, Lithuanian Mathematical Journal, 47(1):48-56, 2007.

7. M.R. Leadbetter, G. Lindgren, and H. Rootzén, Extremes and related properties of random sequences and processes, Volume 11, Springer Verlag, 1983. 
8. Y. Mittal, Comparison technique for highly dependent stationary Gaussian processes, Statistical Extremes and Applications, J.Tiago de Oliveira (ed.). D. Reidel Publishing Company, Holland, pp. 181-195, 1984.

9. Y. Mittal and D. Ylvisaker, Limit distributions for the maxima of stationary Gaussian processes, Stochastic Processes and their Applications, 3(1):1-18, 1975.

10. P. Mladenović and V.I. Piterbarg, On asymptotic distribution of maxima of complete and incomplete samples from stationary sequences, Stochastic Process. Appl., 116(12):1977-1991, 2006.

11. Z. Peng, L. Cao, and S. Nadarajah, Asymptotic distributions of maxima of complete and incomplete samples from multivariate stationary Gaussian sequences, J. Multivariate Anal., 101(10):2641-2647, 2010.

12. V.I. Piterbarg, Asymptotic methods in the theory of Gaussian processes and fields, Volume 148 of Translations of Mathematical Monographs, American Mathematical Society, Providence, RI, 1996.

13. V.I. Piterbarg, Discrete and continuous time extremes of Gaussian processes, Extremes, 7(2):161-177, 2004.

14. Z. Tan and E. Hashorva, Exact tail asymptotics of the supremum of strongly dependent gaussian processes over a random interval, Lithuanian Mathematical Journal, 53(1):91-102, 2013.

15. Z. Tan and E. Hashorva, On Piterbarg max-discretisation theorem for standardised maximum of stationary Gaussian processes, Methodology and Computing in Applied Probability, pp. in press, DOI 10.1007/s11009-012-9305-8, 2013.

16. Z. Tan and Y. Wang, Some asymptotic results on extremes of incomplete samples, Extremes, 15(3):319-332, 2012. 